\title{
Clues in Zodiacal Light Observations for a Dust Ring Along the Earth's Orbit
}

\author{
J.B. Renard (LPCE-CNRS, 3A av. Recherche Scientifique, 45071 Orléans, France) \\ $\mathrm{R}$ Dumont (Observatoire de Bordeaux, 33270 Floirac, France) \\ A.C. Levasseur-Regourd (Paris 6 / Service d'Aéronomie, 91371 Verrières, France) \\ E. Hadamcik (Paris 6 / Service d'Aéronomie, 91371 Verrières, France)
}

\begin{abstract}
The ability of the Earth to trap interplanetary grains into a dust ring lying along the terrestrial orbit was shown by numerical simulations and confirmed by infrared observations (IRAS, COBE). Such a ring could have its signature on the elongation dependence of the zodiacal brightness along the ecliptic, especially near $90^{\circ}$ of the Sun. Indeed, the elongation dependence observed at Tenerife by Dumont and Sanchez (1975) shows that the space density of interplanetary dust slightly increases with increasing heliocentric distance, within the 2 or 3 hundredths of AU approaching Earth's orbit.
\end{abstract}

\section{Introduction}

Numerical simulations (Jackson \& Zook, 1989; Dermott et al., 1994) showed that a fraction of orbitally evolving asteroidal grains could give rise to an «Earth-shepherded» resonant dust ring, in slight excess of density over the main zodiacal cloud near the terrestrial orbit. Confirmations were given with IRAS by Dermott et al. (1994), with COBE by Reach et al. (1995); what was detected, however, is a theoretically expected (Jackson \& Zook, 1992) local inhomogeneity of the ring - a gap in which the Earth is not centered - rather than the whole ring or a wide arc of it.

Wasn't that ring detectable by photometric observations of the zodiacal light ? If its additional density is sufficient, the ring must disturb the smoothness that the elongation-dependence of the brightness should have in the case of a monotonously decreasing density with increasing solar distance. The purpose of the present work is to retrieve from published tables of zodiacal light, clues towards (or against) the existence of the ring.

The elongation-dependence of the zodiacal brightness along the ecliptic, as averaged after the Tenerife campaign (Dumont \& Sanchez, 1975; LevasseurRegourd \& Dumont, 1980), led to determinations of the local scattering coefficient (at right scattering angle), $X$, as a function of heliocentric distance $r$ (Dumont \& Levasseur-Regourd, 1985).

A feature of this $X(r)$ function close to $r=1 \mathrm{AU}$, existing but not focused in the latter papers, is reexamined here. We think that it is the signature of the circumsolar earthbound ring. 


\section{Method of observation}

As shown in previous papers (Dumont, 1983; Dumont \& LevasseurRegourd, 1985), the zodiacal brightness integral $\mathrm{Z}$ (in $\mathrm{S}_{10}(\mathrm{v})$ ) can be written in its simplest form

$$
Z(\varepsilon)=(1 / m) \int_{0}^{\pi-\varepsilon} X(\alpha) d \alpha
$$

0

where $\alpha$ is the phase angle (varying from 0 at $\infty$ to $\pi-\varepsilon$ at the observer's location, with $\varepsilon$ the elongation of the line of sight); $m$ is the subsolar distance of the line of sight (figure 1); $\mathrm{X}$ is the local scattering coefficient in a convenient unit including the intensity of the Sun (i.e. the ultimate information approaching the space density).

Let the line of sight be in the ecliptic, considered as the symmetry plane of the cloud, which is assumed to be cylindrically symmetric. The "secant method" (Dumont, 1973; Dumont \& Levasseur-Regourd, 1985, Reach, 1988) allows us to isolate the brightness contribution of the chord to the terrestrial orbit (considered as circular) .

$$
\Delta Z(E)=Z(\varepsilon)-Z(\pi-\varepsilon)=(1 / \sin \varepsilon) \int_{\varepsilon}^{\pi-\varepsilon} X d \alpha
$$

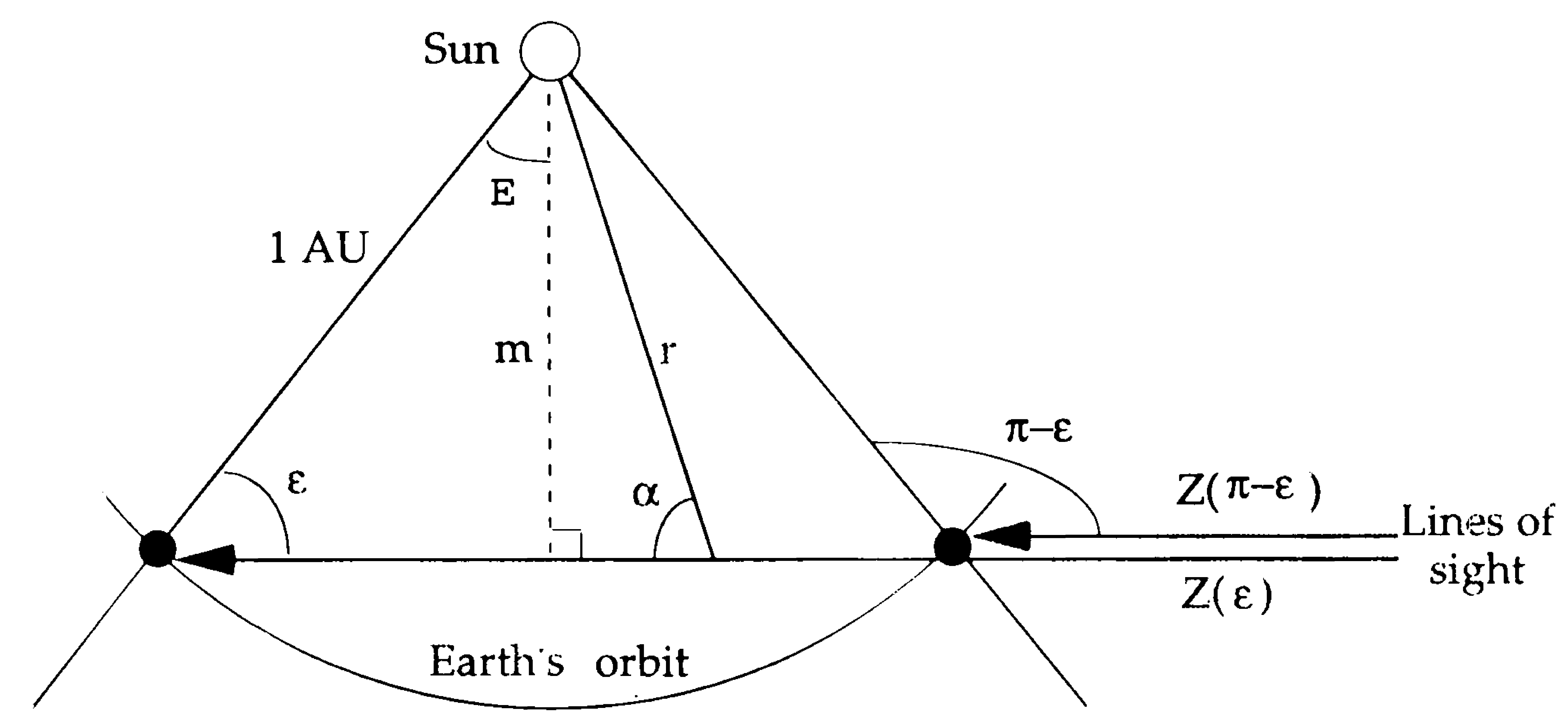

Figure 1 : Geometry of the observations.

\section{Local increase of brightness near $1 \mathrm{AU}$}

Table 1 shows the values of $\Delta Z(E)$ from the Dumont \& Sanchez (1975) observations made at Tenerife (averages of 12 years).

\begin{tabular}{|c||c|c|c|c|c|c|}
\hline $\mathrm{E}$ &, $5^{\circ}$ & $10^{\circ}$ & $15^{\circ}$ & $20^{\circ}$ & $25^{\circ}$ & $30^{\circ}$ \\
\hline \hline$(\varepsilon, \pi-\varepsilon)$ & $\left(85^{\circ}, 95^{\circ}\right)$ & $\left(80^{\circ}, 100^{\circ}\right)$ & $\left(75^{\circ}, 105^{\circ}\right)$ & $\left(70^{\circ}, 110^{\circ}\right)$ & $\left(65^{\circ}, 115^{\circ}\right)$ & $\left(60^{\circ}, 120^{\circ}\right)$ \\
\hline$\Delta \mathrm{Z}(\mathrm{E})$ & 32 & 64 & 98 & 138 & 186 & 247 \\
\hline
\end{tabular}

Table 1 : Values of $\Delta Z(E)$ in $S_{10}(v)$, for various couples of solar elongation. 
Let us assume that the variation of local scattering coefficient with the heliocentric distance can be represented in the $0.866<\mathrm{r}<1.0 \mathrm{AU}$ range by

$$
X(r)=A+B \cdot r+C \cdot r^{2}
$$

where $\mathrm{A}, \mathrm{B}$ and $\mathrm{C}$ are coefficients to be determined.

The geometry of the observations provides the relation $r=\cos E / \sin \alpha$.

Then, after performing the integration on (2), we obtain $\Delta \mathrm{Z}(\mathrm{E})=\mathrm{A} \cdot(2 \mathrm{E} / \cos \mathrm{E})+\mathrm{B} \cdot \log ((1+\sin \mathrm{E}) /(1-\sin \mathrm{E}))+\mathrm{C} \cdot 2 \sin \mathrm{E}$

The solar distance $r_{\min }$ where $X$ is minimum is given by $r_{\min }=-B / 2 C$

To determine $A, B$ and $C, 3$ values of $\Delta Z(E)$ are needed. In order to detect a possible local increase near the Earth's orbit, we must perform the calculations with values not too far from $\mathrm{E}=0^{\circ}$ (typically $5^{\circ}<\mathrm{E}<30^{\circ}$ ).

Figure 2 presents the different curves obtained by using 9 combinations of $\Delta Z(E)$. The results for $r_{\min }, X\left(r_{\min }\right)$ and $X(1 \mathrm{AU})$ are given in table 2.

A minimum for $X(r)$ is found near $r=0.98 \mathrm{AU}$, while $\mathrm{X}(\mathrm{r})$ slightly increases before reaching the Earth's orbit. Such a result shows an increasing density of dust near the Earth's orbit, and the presence of a dust ring, at least $0.02 \mathrm{AU}$ half width.

\begin{tabular}{|c||c|c|c|}
\hline $\mathrm{E}$ & $\mathrm{r}_{\min }(\mathrm{AU})$ & $\mathrm{X}\left(\mathrm{r}_{\min }\right)$ in $\mathrm{S}_{10}(\mathrm{~V}) / \mathrm{rad}$ & $\mathrm{X}(1 \mathrm{AU})$ in $\mathrm{S}_{10}(\mathrm{~V}) / \mathrm{rad}$ \\
\hline \hline $5^{\circ}-10^{\circ}-15^{\circ}$ & 0.981 & 179.4 & 183.7 \\
\hline $5^{\circ}-10^{\circ}-20^{\circ}$ & 0.978 & 179.1 & 183.6 \\
\hline $5^{\circ}-10^{\circ}-25^{\circ}$ & 0.973 & 178.4 & 183.6 \\
\hline $5^{\circ}-10^{\circ}-30^{\circ}$ & 0.967 & 177.6 & 183.5 \\
\hline $5^{\circ}-15^{\circ}-25^{\circ}$ & 0.979 & 179.9 & 183.4 \\
\hline $5^{\circ}-15^{\circ}-25^{\circ}$ & 0.976 & 180.2 & 183.3 \\
\hline $5^{\circ}-15^{\circ}-30^{\circ}$ & 0.974 & 180.3 & 183.2 \\
\hline $10^{\circ}-15^{\circ}-20^{\circ}$ & 0.982 & 179.9 & 182.4 \\
\hline $10^{\circ}-15^{\circ}-25^{\circ}$ & 0.982 & 180.2 & 181.6 \\
\hline \hline Mean value & $0.977 \pm 0.005$ & $179.4 \pm 0.9$ & $183.1 \pm 0.7$ \\
\hline
\end{tabular}

Table 2 : Results for $\mathrm{r}_{\min }, \mathrm{X}\left(\mathrm{r}_{\min }\right)$ and $\mathrm{X}(1 \mathrm{AU})$ obtained by using different combinations of $\Delta \mathrm{Z}(\mathrm{E})$.

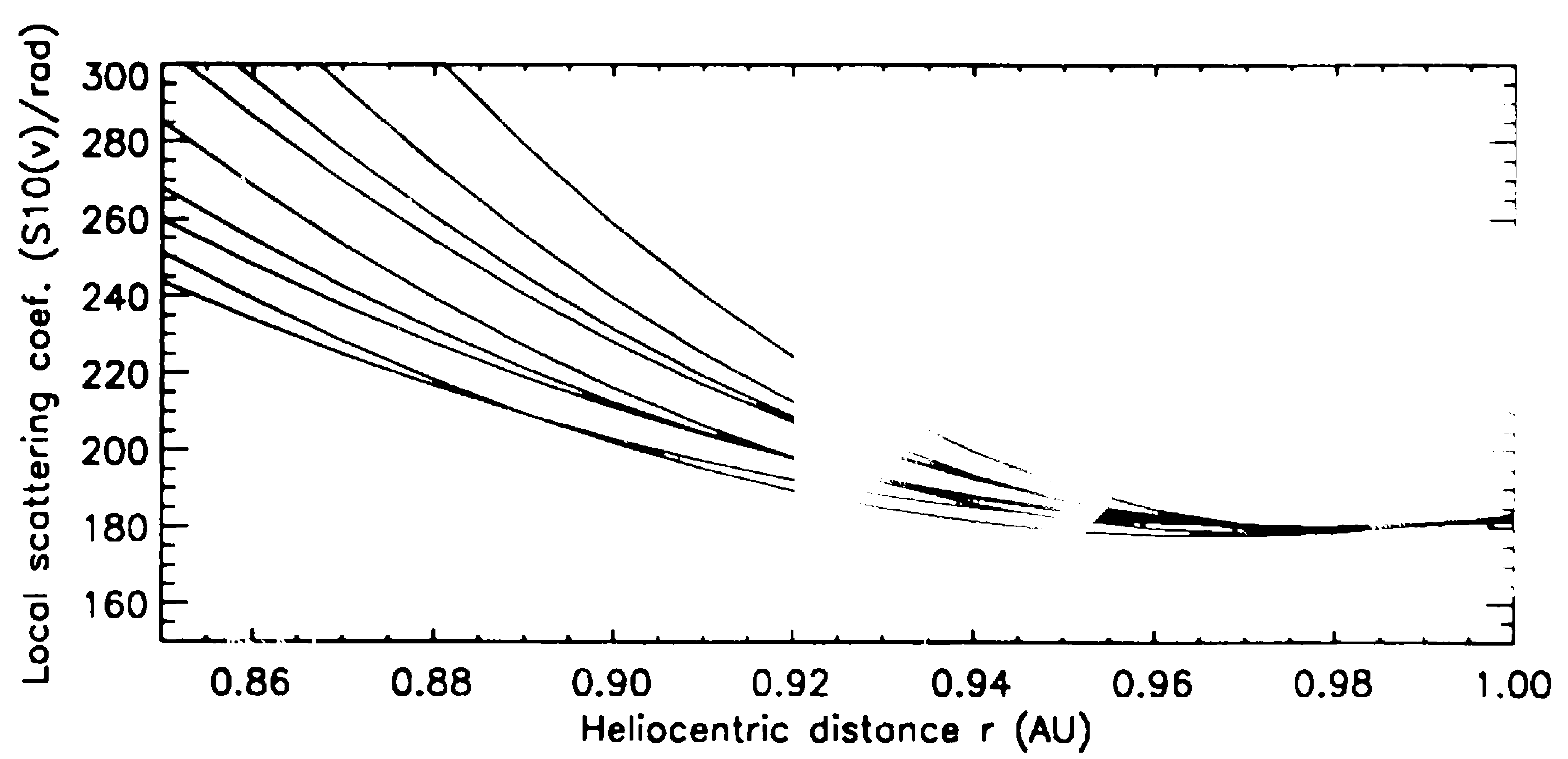

Figure 2 : Evolution of $\mathrm{X}(\mathrm{r})$ for various combinations of $\Delta \mathrm{Z}(\mathrm{E})$. All the curves exhibit a minimum value near $r=0.98 \mathrm{AU}$. 
The «node of lesser uncertainty method», developed by Dumont (1983) \& Dumont and Levasseur-Regourd (1985), provides a straightforward access to the local scattering coefficient $X$, near the centers of the chords to the terrestrial orbit. The radial (heliocentric) dependence of $\mathrm{X}$ has been obtained by Dumont \& Levasseur-Regourd (1985), Levasseur-Regourd et al., (1991) and has been found to follow a power law by Renard (1992), Renard et al., (1995).

Substracting the best power law $\left(\mathrm{r}^{-1.25}\right)$ from the local brightness thus derived in the $0.75<r<1.0$ AU range, a positive residue appears for $r>0.97 \mathrm{AU}$, which we ascribe to the dust ring. At $1 \mathrm{AU}$ the ring adds about $10 \mathrm{~S}_{10}(\mathrm{~V}) / \mathrm{rad}$ to the scattering coefficient of the main zodiacal cloud.

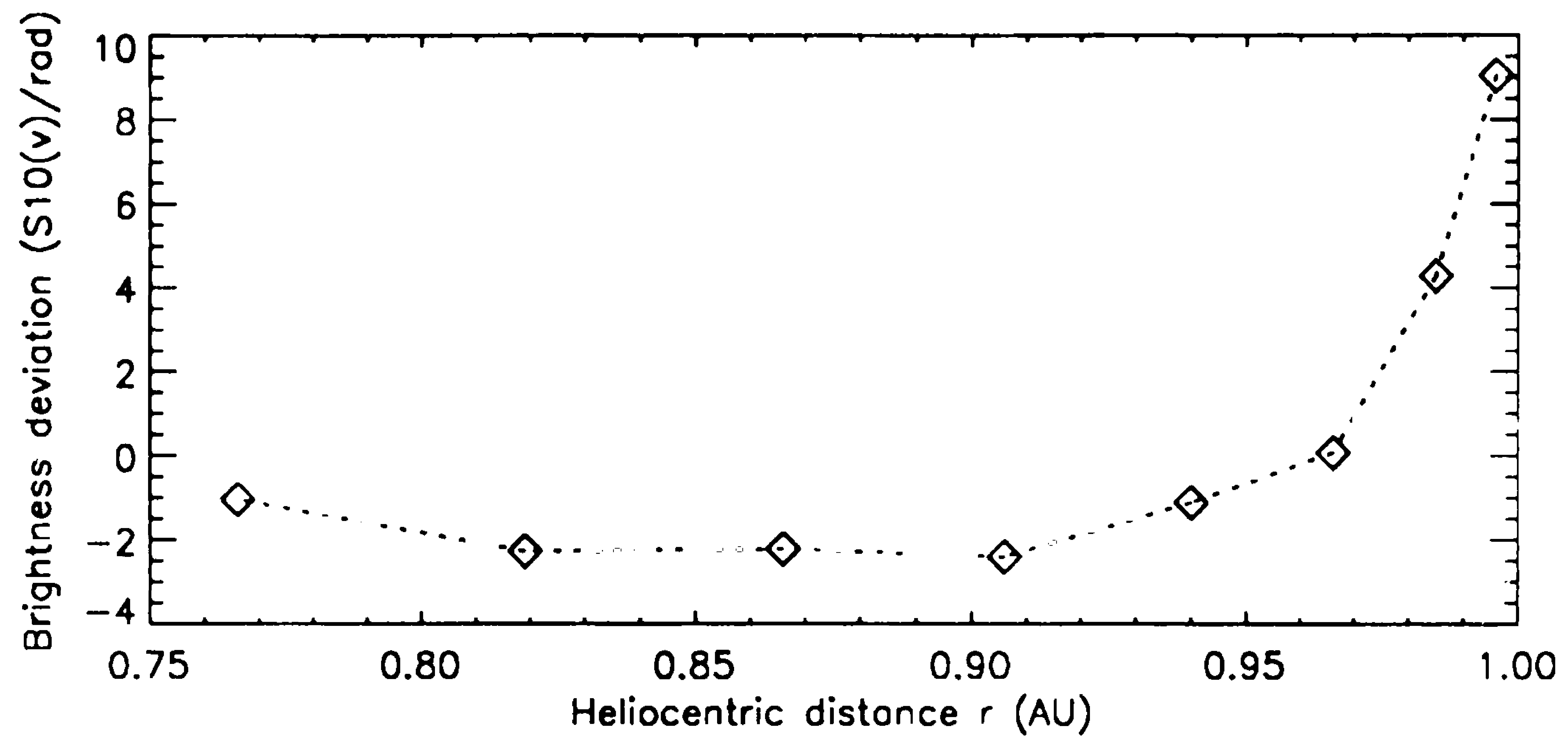

Figure 3 : Deviation from the power law which represents the evolution of the local brightness. The increase for $r>0.97 \mathrm{AU}$ is ascribed to the dust ring.

\section{Conclusion}

By using two different approaches that consider the local brightness of the zodiacal light, an increase is found near $1 \mathrm{AU}$. In our opinion, this increase can signal the presence of a dust ring around the Earth's orbit. Because the geometry of observations allows us to retrieve the brightness for solar distance $r<1 \mathrm{AU}$, only the inner side of the ring is detected. Its half width is of the order of 0.03 $\mathrm{AU}$, and its brightness excess is about $10 \mathrm{~S}_{10}(\mathrm{~V}) / \mathrm{rad}$, which represents $1 / 20$ of the zouiacal cloud local brightness at the Earth's orbit.

Thus, Tenerife photometry of the ecliptic, published 20 years ago, therefore contains a clue towards the existence of the Earth-orbit ring, with some information about its additional space density and about its half-width.

\section{References}

Dermott S.F et al., Nature 369, 6483, 719, 1994.

Dumont R., Planet. Space Sci. 21, 2149, 1973.

Dumont R., Planet. Space Sci. 31, 1381, 1983.

Dumont R., Sanchez F , Astron. Astrophys. 38, 405, 1975.

Dumont R., Levasseur-Regourd A.C., Planet. Space Sci. 33, 1, 1985.

Jackson A.A., Zook H.A., Nature 337, 629, 1989.

Jackson A.A., Zook H.A., Lunar and Planet. Sci. 23, 595, 1992.

Levasseur-Regourd, A.C., Dumont, R., Astron. Astrophys. 84, 277, 1980.

Levasseur-Regourd A.C., et al., Adv. Space Res. 11, 12, 175, 1991.

Renard J.B., Thesis, University of Paris 6, 1992.

Renard J.B., et al., Astron. Astrophys., in press, 1995.

Reach, W T , Astrophys. J. 335, 468, 1988.

Reach et al., Nature 374, 521, 1995. 